\title{
Noncanonical E2 recruitment by the autophagy E1 revealed by Atg7-Atg3 and Atg7-Atg10 structures
}

\author{
Stephen E. Kaiser ${ }^{1}$, Kai Mao ${ }^{2}$, Asad M. Taherbhoy ${ }^{1,3}$, Shanshan $\mathrm{Yu}^{1,3}$, Jennifer L. \\ Olszewski ${ }^{1}$, David M. Duda ${ }^{1,4}$, Igor Kurinov ${ }^{5}$, Alan Deng ${ }^{1}$, Timothy D. Fenn ${ }^{6}$, Daniel J. \\ Klionsky ${ }^{2}$, and Brenda A. Schulman ${ }^{1,4}$ \\ ${ }^{1}$ Department of Structural Biology, St. Jude Children's Research Hospital, Memphis, Tennessee, \\ USA
}

${ }^{2}$ Life Sciences Institute; University of Michigan; Ann Arbor, MI USA

${ }^{3}$ Integrated Program in Biomedical Sciences, University of Tennessee Health Science Center, Memphis, Tennessee, USA

${ }^{4}$ Howard Hughes Medical Institute, St. Jude Children's Research Hospital, Memphis, Tennessee, USA

${ }^{5}$ Department of Chemistry and Chemical Biology, Cornell University, Argonne, IL 60439

${ }^{6}$ Department of Bioengineering, Stanford University, Stanford, CA, USA

\section{Abstract}

Core functions of autophagy are mediated by ubiquitin-like protein (UBL) cascades, in which a homodimeric E1 enzyme, Atg7, directs the UBLs Atg8 and Atg12 to their respective E2 enzymes, $\operatorname{Atg} 3$ and Atg10. Crystallographic and mutational analyses of yeast $(\operatorname{Atg} 7-\operatorname{Atg} 3)_{2}$ and $(\operatorname{Atg} 7-$ Atg 10) 2 complexes reveal noncanonical, multisite E1 -E2 recognition in autophagy. Atg7's unique N-terminal domain recruits distinctive elements from the Atg3 and Atg10 'backsides'. This, along with E1 and E2 conformational variability, allows presentation of 'frontside' Atg3 and Atg10 active sites to the catalytic cysteine in the C-terminal domain from the opposite Atg7 protomer in the homodimer. Despite different modes of binding, the data suggest that common principles underlie conjugation in both noncanonical and canonical UBL cascades, whereby flexibly tethered E1 domains recruit E2s through surfaces remote from their active sites to juxtapose the E1 and E2 catalytic cysteines.

\footnotetext{
Users may view, print, copy, download and text and data- mine the content in such documents, for the purposes of academic research, subject always to the full Conditions of use: http://www.nature.com/authors/editorial_policies/license.html\#terms

Correspondence: Brenda A. Schulman, St. Jude Children's Research Hospital, 262 Danny Thomas Place, MS\#311, Memphis, TN 38105, Phone: 901-595-5147, brenda.schulman@stjude.org.

Author contributions: SK, JO, DMD, IK, and TDF performed the x-ray crystallography experiments. SK, AMT, SY, JO, and AD purified wild-type and mutant proteins and performed in vitro biochemical assays. KM performed all yeast experiments. BAS, along with SK and DJK, oversaw experiments, and prepared the manuscript with input from all authors.

Competing financial interests: None of the authors has competing financial interests.

Accession codes: Structure factor data and coordinates for Atg7NTD K14A F16A D18A, Atg7-Atg10, and Atg7-Atg3 have been deposited to the RCSB with accession codes 4GSJ, 4GSK, and 4GSL, respectively.
} 


\section{Introduction}

In the eukaryotic process of autophagy, or 'self-eating', a double-membrane phagophore, the precursor to an autophagosome, engulfs a portion of the cytoplasm and the latter then fuses with lysosomes or the vacuole for degradation of the cytosolic contents. This can allow recycling of macromolecules when cells are starved of nutrients, or elimination of defective or unneeded organelles or cytoplasmic assemblies.

Core functions of autophagy are mediated by noncanonical ubiquitin-like protein (UBL) cascades. Best-characterized in the yeast Saccharomyces cerevisiae, the two UBLs Atg8 and Atg12 are both activated by the same E1 enzyme, Atg7, which by mechanisms that are not well understood, directs each UBL to an E2 enzyme, Atg3 or Atg10, respectively ${ }^{1-3}$. Ultimately, the Atg7 and Atg3 cascade ligates Atg8's C-terminal Gly to phosphatidylethanolamine (PE). Here a tilde $(\sim)$ denotes thioester- or peptide-bonded covalent complexes. Atg8 PE adducts are incorporated into the growing autophagosomal membrane in a process that is critical for its expansion, and Atg8 also recruits cargo during selective autophagy ${ }^{4,5}$. The other autophagic UBL, Atg12, functions after Atg7 and Atg10mediated ligation to Atg5 to enhance Atg8 lipidation ${ }^{6-11}$. The importance of Atg7, Atg3, Atg10, and other components of the Atg8 and Atg12 ligation pathways is reflected by numerous pathologies observed in cells, tissues, and/or animals lacking these proteins ${ }^{12-15}$. Thus, it is of great significance to understand molecular mechanisms underlying UBL conjugation in autophagy.

Enzymes in the Atg8 and Atg12 conjugation cascades are termed 'noncanonical' due to substantial differences from those for UBLs such as ubiquitin, NEDD8, and SUMO ${ }^{16}$. Unlike progress in understanding the structural characteristics of enzymes involved in canonical UBL conjugation cascades, our knowledge of the noncanonical features underlying Atg8 and Atg12 conjugation remains relatively rudimentary. Briefly, in canonical UBL cascades, as well as noncanonical cascades in autophagy, an E1's structurally conserved adenylation domain binds the UBL and catalyzes its C-terminal adenylation ${ }^{17-20}$. However, in Atg7, the adenylation domain is a symmetric homodimer, with two identical UBL-binding and adenylation active sites, whereas the adenylation domains from canonical E1s are pseudosymmetric and contain only a single UBL binding and adenylation active site ${ }^{21-23}$. The UBL C terminus subsequently becomes covalently attached to the E1 catalytic cysteine via a thioester bond. In canonical E1s, the catalytic cysteine is housed in a distinct domain ${ }^{18}$, which undergoes remarkable structural remodeling to form an E1 UBL thioester intermediate ${ }^{24}$. By contrast, Atg7's cysteine is in a flexible loop that crosses over the UBL-binding surface and approaches an autophagy UBL's Cterminal Gly in the adenylation active site ${ }^{21-23}$. Thus, structurally, Atg7 has a single Cterminal domain (CTD) that contains the elements both for UBL adenylation and forming the Atg7 UBL thioester intermediate. Next, a UBL thioester is transferred to an E2 catalytic cysteine, in order to ultimately shuttle the activated UBL to targets. However, the mechanisms by which canonical and noncanonical E1s recruit their E2s differ completely. Canonical E1s recruit their cognate E2s via a selective 'ubiquitin-fold domain' (ufd) at the C terminus of the same subunit that also harbors the adenylation active site and catalytic cysteine ${ }^{16,25,26}$. By contrast, Atg7 lacks a ufd, and instead has a unique 'N-terminal domain' 
(NTD) that binds autophagic E2s ${ }^{21-23}$. For Atg3 this was shown to utilize a unique 'trans' architecture: Atg3 binds the NTD of one Atg7, and receives Atg8 from the catalytic cysteine of the opposite Atg7 molecule in the homodimer ${ }^{21-23}$.

The autophagy E2s also have distinctive features that raise questions about how both Atg3 and Atg10 could bind Atg7. First, Atg3 and Atg10 share little sequence homology. Although they both have core structures resembling a canonical E2 catalytic domain, with a concave $\beta$-sheet 'backside' and the catalytic cysteine at the 'front', their mechanisms of E1 binding are apparently noncanonical ${ }^{27,28}$. Atg3 lacks any known catalytic residues other than the active site cysteine, and also has a unique 'flexible region' (FR) that binds Atg7 and a distinctive 'handle region' (HR) required for downstream steps in Atg8 lipidation 27 . A prior structure of a short peptide from the Atg3 FR bound to the isolated Atg7 NTD revealed the basis for their high affinity interactions, although additional contact surfaces were implied ${ }^{21}$. Also, it is unclear how Atg10 binds Atg7: Atg10 lacks an obvious FR sequence, and an Atg7 mutant at the Atg3FR-binding interface is defective for the Atg3 pathway but not for the Atg10 pathway in vivo ${ }^{21}$. Seemingly paradoxically, the isolated Atg3FR competes with Atg10 for Atg7-binding in vitro, and the Atg7 binding site on Atg3FR is not accessible in the prior structure of full-length $\operatorname{Atg} 3^{23,27}$.

Thus, to gain insights into how the autophagy E1 recognizes its E2s, we determined crystal structures of Atg7-Atg3 and Atg7-Atg10 complexes from S. cerevisiae. The structures show how a single E1 enzyme uses the same surfaces to recognize distinctive features of two different E2s, and how conformational changes and unique molecular architectures mediate active site juxtaposition for the noncanonical E1-E2 complexes in autophagy.

\section{Results}

\section{Multisite recruitment of Atg3 and Atg10 to Atg7}

To visualize the juxtaposition of E1 and E2 catalytic cysteines, which is required for UBL transfer, we performed crosslinking as follows: we purified versions of Atg3 and Atg10 containing only a single cysteine at the active site, reacted the homobifunctional sulfhydryl crosslinker bismaleimidoethane (BMOE) with each E2, and desalted excess crosslinker. For Atg3, we added a version of Atg7 lacking the C-terminal 17-residues disordered in prior crystals, and for Atg10 we added a version of Atg7 with this truncation and C39S C195S C375A mutations to reduce background crosslinking. Using this method, we obtained crystal structures of Atg7-BMOE-Atg3 and Atg7-BMOE-Atg10 (hereafter referred to as Atg7-Atg3 and Atg7-Atg10) to 2.7 and $2.9 \AA$ resolution, respectively (Table 1 and Supplementary Fig. 1). Both contain one dimeric Atg7 bound to two E2s per asymmetric unit.

The structure of Atg7 resembles a bird, with the two NTDs corresponding to 'wings' extending away from the symmetric, homodimeric CTD 'body' (Fig. 1a and Supplementary Fig. 2a). The relative NTD-CTD orientations differ by $\sim 10^{\circ}$ between all the E2-bound and the unbound Atg7 structures ${ }^{22}$, translating into $\sim 40 \AA$ differences in the distal edge positions for the oblong NTD. The range of NTD positions observed upon superimposing the E2bound and apo Atg7 structures over their CTDs looks like a bird flapping its wings. 
Conformational flexibility between the Atg7 NTD and CTD is likely important to accommodate the different dimensions of the Atg3 and Atg10 E2s, and is consistent with prior small angle $\mathrm{X}$-ray scattering data ${ }^{21,22}$.

The E2 core domain of Atg7-bound Atg3 superimposes well with the prior uncomplexed structure $^{27}$, although in the complex the Atg3 catalytic cysteine loop is substantially rearranged, and much of the HR is not visible (Fig. 1b). In both the free and Atg7-bound Atg3 structures, the majority of the FR is disordered, except for a small helical segment. However, this region of the Atg3FR is substantially displaced in the complex with Atg7: instead of packing against Atg3's own E2 core domain, the FR helix interacts with a distal groove in the 'shoulder' region of the Atg7 NTD 'wing'.

Despite little apparent overall sequence similarity, Atg10 also generally resembles a canonical E2 core domain. Although the C-terminal structures differ between Atg3, Atg10, and canonical E2s, their catalytic domains share in common an N-terminal helix, a 'backside' 4-stranded antiparallel $\beta$-sheet followed by the cysteine loop, and a long central helix ${ }^{28}$ (Fig. 1b). One notable difference in Atg10 when compared to Atg3 and other E2s is C-terminal extension of the $4^{\text {th }} \beta$-strand and its continuation into a $\beta$-hairpin that leads to an additional strand incorporated into an extended $\beta$-sheet on the E2 'backside' (Fig. 1b).

Common Atg7 surfaces recruit the E2s through extensive, multipart interfaces involving (1) the 'shoulder' portion of the Atg7 NTD 'wing' interacting with unique structures from the backs of Atg3 and of Atg10, (2) the 'under wing' portion of the NTD interacting loosely with features along the length of the backsides of both E2s, and (3) the Atg7 NTD-CTD junction cradling the edge of both E2s (Fig. 2a, 2b). Atg7's multisite recruitment buries $\sim 2,450$ and $\sim 1,830 \AA^{2}$ from Atg3 or Atg10, respectively, which corresponds to $\sim 30 \%$ of their exposed surfaces.

The structurally observed Atg7 NTD recruitment of an autophagic E2 via its backside allows juxtapositioning of the E2 'frontside' active site with the catalytic cysteine in the CTD of the opposite Atg7 molecule in the homodimer (Fig. 2). This trans configuration agrees with previous biochemical studies of Atg7-Atg3 complexes. To test whether this trans architecture exists in solution also for Atg7-Atg10 complexes, we performed crosslinking based on previously described mixed dimer versions of Atg $7^{21,22}$. In the 'trans' configuration, one Atg7 molecule consists only of the CTD, which retains the catalytic cysteine but lacks the E2-binding NTD. The other Atg7 retains the E2-binding NTD, but carries an active site cysteine-to-alanine substitution in the CTD. In the 'cis' configuration, the Atg7 CTD harbors a catalytic cysteine-to-alanine mutation, and the full-length Atg7 retains both the E2-binding NTD and the catalytic cysteine. Mixed heterodimers are stabilized by an engineered salt-bridge between glutamate and arginine substitutions in place of residues 511 and 524, respectively ${ }^{22}$, and are ensured via a sequential 2-tag purification strategy ${ }^{21}$. After reacting single cysteine versions of either Atg3 or Atg10 with BMOE, desalting, and adding one of the two forms of Atg7, crosslinking is observed only with the 'trans' version of Atg7, consistent with the crystallographic architectures (Fig. 2c). 


\section{E2 recruitment to a conserved Atg7 NTD shoulder groove}

An $~ 30 \AA$ long, conserved hydrophobic groove in the 'shoulder' region of the Atg7 NTD 'wing' anchors the two autophagy E2s in completely different ways. From Atg3, the unique FR helix is embedded in the portion of the shoulder groove lined by L90, F93, K94, K98, R135, Y137, W139, W273, K280, L281, P283 and V285 from the Atg7NTD (Fig. 3a), as in the prior structure of the isolated Atg7NTD bound to a short Atg3FR helix peptide ${ }^{21}$. Although the 45 residues preceding the FR helix are disordered in the Atg7-Atg3 complex, there is patchy electron density corresponding to the residues following the helix. Notably, this un-modeled density traverses a basic surface in the middle of Atg7's NTD, likely reflecting electrostatic interactions from up to 12 of the 20 residues in this region that are acidic or polar (Supplementary Figs. 3, 4). The importance of Atg7's shoulder carrying Atg3's FR is highlighted by the previous finding that deleting the FR increases the Kd for Atg7 binding substantially ${ }^{21}$.

From Atg10, the $\beta$-hairpin binds the edge of the shoulder groove (Fig. 3b). Here, Atg10's Ile88, Asp89, Ile91, and Pro92 are enwrapped in a pocket formed by Atg7's R135, Y137, N276, V277, Q278, A282, P283, V285, and D287. The interaction is stabilized by saltbridges between Atg7's Lys94 and Atg10's Asp89, and between Atg7's Asp287 and Ser290 and Atg10's Lys95. Strikingly, the additional $\beta$-strand in Atg10's sheet aligns with Atg7's $\beta$ strand 15. This leads to formation of a 10-stranded interprotein $\beta$-sheet, with 5 strands each from Atg10's backside and Atg7's NTD.

Comparison of Atg3 and Atg10 contacts with the 'shoulder' groove resolves previous seemingly contradictory findings. Some overlap of the interaction surfaces (Fig. 3c) explains why the Atg3FR competed with Atg10 for binding to the Atg7NTD during gel filtration ${ }^{23}$. Although we wished to further probe the competition, unfortunately, we have not been able to purify S. cerevisiae Atg12. Thus, we developed an artificial enzyme assay: using high protein concentrations in vitro, we can observe non-native Atg7-mediated conjugation of Atg8 onto Atg10 at low levels. This reaction is inhibited by the short peptide corresponding to the Atg3FR helix (Fig. 3d), consistent with the structures.

Despite overlapping binding surfaces, the distinct E2 binding modes suggest that different Atg7 residues from the shoulder groove play critical roles in recruiting Atg3 or Atg10. In particular, the Pro283 side-chain is central to the hydrophobic interaction with Atg3FR, but is at the edge of the Atg10-binding surface. By contrast, Val285 is at the center of the hydrophobic interface with the Atg10 $\beta$-hairpin, but is on the edge of the Atg3-binding surface. At a more intermediate level, Tyr137 also plays a modest role in the Atg3 interaction, but appears to stabilize the structure of the Atg10-binding site. We tested these concepts with Atg7-E2 catalytic cysteine crosslinking assays (Fig. 3e, f). In agreement with the structures, mutation of Atg7's Tyr137 has modest effects on crosslinking to both E2s. However, a P283D substitution shown previously to impair Atg7-Atg3 interactions has little affect on Atg10 crosslinking to Atg7, whereas a V285D substitution almost abolished Atg7 crosslinking to Atg10 but not to Atg3. Deleting Atg10's $\beta$-hairpin (residues 86-93) also substantially diminished crosslinking to Atg7 (Fig. 3g). Thus, the Atg7 shoulder groove is 
built to recruit distinct distal structures from the two E2s in such a way as to allow each of their catalytic centers to approach the Atg7 active site.

\section{E2 positioning through backside and edge binding to Atg7}

Although prior qualitative studies suggested that Atg3's FR is essential for binding to $\operatorname{Atg} 7^{27}$, additional contacts were implicated from the findings that Atg7 binds weakly to Atg3 deleted for the FR region, that Atg7's NTD binds with higher affinity to full-length Atg3 than to a peptide corresponding to Atg3's FR helix, and that Atg3 binds with higher affinity to full-length Atg7 than to the isolated Atg7 NTD ${ }^{21}$. Indeed, more than $30 \operatorname{Atg} 7$ 'underwing' residues make more than 140 contacts to more than 25 residues from all four strands corresponding to the E2 core domain backside of Atg3, as well as to several additional Atg3-specific loops (Fig. 4a, Supplementary Fig. 5). Here, Atg7 and Atg3 interact through three regions that together comprise a roughly circular surface with an $\sim 30 \AA$ diameter. At the heart is a hydrophobic cluster involving Atg7's Phe16 and Phe61 and Atg3's Tyr168 and Phe189, buttressed by Atg7's Lys14 and Asp18, and backside surface side-chains from Atg3 including Ser54, Arg72, Lys73, Asp170 and the backbone oxygen from Ser191.

We tested roles of this extensive, secondary Atg7-Atg3 binding surface using several assays. To probe interactions, we used our Atg7-Atg3 catalytic cysteine crosslinking assay, and to probe enzymatic activity, we examined in vitro pulse-chase transfer of ${ }^{32} \mathrm{P}$-Atg8 from Atg7 to Atg3. Consistent with key roles in stabilizing Atg7-Atg3 interactions and positioning their active sites, multiple Ala substitutions in place of Atg7 or Atg3 surface residues at the hydrophobic center of this interface result in severe defects in both in vitro assays (Fig. 4b-d). For the highly deleterious K14A F16A D18A mutant, we confirmed proper folding with a crystal structure of this mutant version of Atg7NTD (Supplementary Fig. 2b, Table 1). By contrast, mutations at the distal edge- Atg7 D47A N50A K53A or Atg3 K48A E51A Q302A D304A—had little effect on Atg7-Atg3 interactions in vitro.

To extend our analysis and study the effects of these Atg7 and Atg3 mutants in vivo, we examined their autophagy activity by four established methods: GFP-Atg8 localization, Pho8 600 and GFP-Atg8 processing assays and an Atg8 lipidation assay (Fig. 4e,f, Supplementary Fig. 6). During autophagy, GFP-Atg8 becomes ligated to PE and becomes localized to punctate dots, whereas it remains diffusely localized in the cytoplasm in yeast strains deficient in Atg8 lipidation. The second assay relies on Pho8 $\Delta 60$, a mutant form of vacuolar alkaline phosphatase with $60 \mathrm{~N}$-terminal amino acid residues deleted, normally remaining in the cytosol, and transported into the vacuole only through autophagy ${ }^{29}$. Measuring the activity of alkaline phosphatase thus reflects the level of autophagy. In the third assay, GFP-Atg8 is delivered into the vacuole when autophagy is induced. Although Atg8 is degraded, the GFP moiety is relatively stable. Therefore, autophagy progression can be determined by the accumulation of free $\mathrm{GFP}^{30}$. Finally, we monitored Atg8 lipidation as a mobility shift on SDS-PAGE gels. Wild-type but not atg $7 \Delta$ or atg $3 \Delta$ cells transformed with empty vector displayed autophagy as monitored by all four assays. Autophagy was restored in $\operatorname{atg} 7 \Delta$ cells with a plasmid expressing either wild-type Atg7 or Atg7D47A N50A $\mathrm{K} 53 \mathrm{~A}$, and in atg $3 \Delta$ cells expressing wild-type Atg3. In contrast, autophagy was abolished 
in atg7 $\Delta$ cells expressing Atg7 K14A F16A D18A or Atg7 F16A F61A, or in atg3 $\Delta$ cells expressing Atg3 R72A K73A Y168A, all of which contain mutations that localize to the center of the hydrophobic interface.

We were surprised to find that although Atg3 K48A E51A Q302A D304A with mutations at the distal edge showed little defect in Atg8 transfer from Atg7 to Atg3 in our in vitro assays, these mutations severely disrupted autophagy (Fig. 4b, 4f, Supplementary Fig. 6). Thus, it seemed possible that Atg3 uses this surface for downstream interactions in the pathway. Accordingly, we examined Atg8 lipidation with an in vitro assay in which Atg12 Atg5 acts as an E3 and PE-containing liposomes provide substrate (Fig 4g, 4h). Consistent with the in vivo data, this mutant version of Atg3 is defective for generating the Atg8 PE product in vitro, suggesting potential roles for this surface in binding to $\mathrm{E} 3$ or substrate.

The Atg7 NTD-CTD junction region is also functionally important, as deleting Atg7 residues 290-294 or inserting the flexible sequence Gly-Gly-Ser-Gly after Atg7's Leu291 leads to decreased crosslinking with Atg3 (Fig. 4d,i). The insertion mutation was shown previously to decrease Atg7-mediated ${ }^{32} \mathrm{P}$-Atg8 transfer to $\operatorname{Atg} 3^{21}$, and here we also observe similar defects for the deletion (Fig. 4j).

The Atg7 underwing region also interacts with Atg10's backside and edge (Fig. 5a). However, unlike interactions with Atg3, Atg7's 'underwing' contacts to Atg10 are dispersed and lack a distinct center, and thus may arise from crystallization or conformations imposed by crosslinking. Accordingly, multiple Ala substitutions in the underwing region of Atg7 had only a minor affect on crosslinking to Atg10 (Fig. 5b). Furthermore, aligning either the Atg7 NTDs or the E2s from the Atg7-Atg3 and Atg7-Atg10 complexes reveals relative displacement for the structural elements mediating interactions, and different relative Atg7 NTD and CTD orientations for juxtaposition of Atg7's active site with those of the two E2s (Fig. 1a, 2a, 2b, 5c).

At the near edge, Ser290 and Leu291 from Atg7's NTD-CTD junction interact with Atg10's Leu96 and Leu98, with continued interactions involving the N-terminal helix of Atg7's NTD. Here, Atg7's Gln300 and Lys307 contact the backbone oxygens of Leu98 and Pro99, and Glu103 from Atg10. Additional electron density connecting Atg10's Val108 and Gly124 that is too low quality for accurate modeling reflects further contact with Atg7's NTD-CTD junction. Indeed, either deleting or inserting residues at the Atg7 NTD-CTD junction resulted in decreased crosslinking to Atg10 (Fig. 5b).

\section{Atg7-binding unmasks conserved E2 active site architectures}

For both Atg3 and Atg10, the E2 active site is on the opposite face from the noncovalent Atg7 binding regions. In complex with Atg7, Atg3's catalytic Cys234 is surface exposed, and is connected to additional patchy electron density that presumably corresponds to the BMOE also linked to Atg7's catalytic Cys507 7 A away. Here, Atg3's cysteine-loop is substantially rearranged from the conformation observed in free Atg3, in which Cys 234 is buried $^{27}$ (Fig. 6a). Atg10's catalytic Cys133 is not observed in either complex in the asymmetric unit, suggesting that Atg10's cysteine-loop is also conformationally flexible. Nonetheless, the location of Atg10's Cys133 can be approximated based on the positions of 
His131 visible in one Atg10 and Pro132 in the other Atg10 in the assymetric unit. These are consistent with the subsequent Cys133 residue approaching Atg7's Cys507.

Despite Atg10's catalytic cysteine not being visible and the limited resolution of our data, comparison of the Atg3 and Atg10 structures suggest a common active site architecture: Atg3's catalytic cysteine is flanked by Tyr179 and His232, and Atg10's Tyr73 and His131 are in parallel locations (Fig. 6b). Notably, these are among the few amino acids shared between the sequences of Atg3s and Atg10s, suggesting key functions (Supplementary Fig. 4). Indeed, Atg3 Y179A decreases pulse-chase transfer of ${ }^{32} \mathrm{P}-\mathrm{Atg} 8$ from Atg7 to Atg3, and both mutants are defective in Atg8 lipidation in vitro, with a more pronounced defect observed for H232A (Fig. 6c, 6d). Notably, these catalytic residues are required for Atg3 and Atg10 function in vivo (Fig. 6e, 6f).

The conformation of the Atg7 cysteine loop when crosslinked to the E2s also differs from previous structures ${ }^{21-23}$. Prior studies had revealed that in the absence of a UBL, the Atg7 cysteine loop packs closely against the UBL-binding surface, with Cys507 facing toward the E1. However, in complex with Atg8, Atg7's cysteine loop is separated from the CTD and crosses over Atg8's C terminus. Interestingly, in different crystals of Atg7CTD-Atg8 complexes, Atg7's residues 504-508 are observed in different conformations, with Cys507 facing either toward or away from Atg8's C-terminal Gly ${ }^{22,23}$. When crosslinked to the E2s but in the absence of a UBL, Atg7's cysteine loop adopts a hybrid conformation, with residues 490-505 packing against Atg7's UBL binding site as observed previously, and 506-508 more open to allow Cys507 to face an E2 active site (Fig. 6g).

\section{Discussion}

The Atg7-Atg3 and Atg7-Atg10 crystal structures have revealed how the noncanonocal E1, Atg7, recognizes its two cognate but distinct E2s. Both Atg3 and Atg10 are recruited via their E2 core domain backsides to a broad surface extending over much of the unique Atg7 NTD and including the junction with the Atg7 CTD. Notably, Atg3- and Atg10-specific insertions anchor the complexes through docking in a distal Atg7 NTD groove. Atg7 NTDmediated E2 backside recruitment, coupled with conformational flexibility, allows presentation of the active sites on the front sides of Atg3 and Atg10 to the remote catalytic cysteine in the UBL-binding CTD from the opposite Atg7 monomer in the homodimer.

In addition to functioning with these two divergent E2s, Atg7 is distinct among E1s in activating two divergent UBLs, Atg8 and Atg12. It is currently thought that Atg8 is strictly directed to Atg3 as its specific $\mathrm{E} 2$ for ligation to $\mathrm{PE}^{31}$, with Atg 10 being the exclusive $\mathrm{E} 2$ for Atg12 ligation to $\operatorname{Atg} 5^{32}$. However, there is much crosstalk between the pathways. For example, the Atg12 Atg5 complex is an E3 that promotes Atg8 transfer from Atg3's active site to $\mathrm{PE}^{6}$. Conversely, in mammalian cells, Atg3 overexpression facilitates formation of the Atg12 Atg5 complex ${ }^{33}$, and during mitophagy Atg3 can also act as an Atg12 E2 mediating self-modification ${ }^{34}$. Irrespective of whether there are circumstances under which Atg8 and Atg12 could both function with Atg3 and/or Atg10, it is clear that, at least in vitro, Atg8 is preferentially transferred to Atg3. 
Two speculative models emerge for how Atg7 UBL and E2 specificity could be established. Briefly, we made a model of Atg3-Atg7 Atg8 by superimposing the Atg7-Atg3 structure with a prior Atg7CTD-Atg8 structure ${ }^{23}$, and (1) docked the Atg8 globular domain, (2) transplanted residues 490-506 from the Atg7 cysteine loop in the Atg8-bound complex, and (3) positioned Atg8's C-terminal tail as in a thioester complex by superimposing them on Atg7's cysteine loop residues 504-506 from the structures lacking Atg8 (Fig. 7a). The structural model reveals the Atg7 cysteine loop contacting both Atg8 and Atg3. Thus, the Atg7 cysteine loop may be the sensor that transmits signals between the UBL and the E2 for optimal interaction and/or catalysis of UBL transfer. The second mechanism is suggested by a parallel model of Atg10-Atg7 Atg12 (Fig. 7b). Here, Atg10's residues 133-145, including Cys133, are not modeled because they were not visible in the electron density. However, the locations of the adjoining Atg10 residues Pro132 and Met146 suggest that the E2 cysteine loop may also directly recognize its cognate UBL during the transthiolation reaction. Future structural studies of Atg3-Atg7 Atg8 and Atg10-Atg7 Atg12 complexes will be required to understand the extent to which the E1 and E2 cysteine loops or other features dictate UBL specificity of E1-to-E2 transfer in autophagy.

In addition to implications for autophagy, our data also broadly show how one E1 uses the same surfaces to bind distinctive elements from 2 different E2s. Although the mechanisms by which Atg7 recruits Atg3 and Atg10 differ from those by which canonical E1 ufds recruit E2s (Supplementary Fig. 7), some common principles emerge: in all cases, E2s are anchored to E1 domains through surfaces remote from the active sites, with the E1 domains likely rotating to bring the E1 and E2 catalytic cysteines into proximity for catalysis ${ }^{16,25}$. Furthermore, Atg3 and Atg10 now join a long list of E2s for which key interactions involve the 'backside' $\beta$-sheet ${ }^{35}$ (Supplementary Fig. 7). Many E2s for ubiquitin, and also the SUMO E2 Ubc 9 ${ }^{36-38}$, 'backside bind' noncovalently to their cognate UBL. This can promote polyUBL chain synthesis through unknown mechanisms ${ }^{35,38}$. For the ubiquitin E2 Ube2g2, backside binding to a helix from the $\mathrm{E} 3 \mathrm{gp} 78$ allosterically promotes polyubiquitin chain synthesis ${ }^{39,40}$. Interestingly, the E2 Rad6 can backside bind in a mutually exclusive manner to ubiquitin or to a helix from the E3 Rad8, the latter restraining activity to monoubiquitination ${ }^{41}$. As with ubiquitin and SUMO E2s, Atg3 and Atg10 make numerous protein-protein interactions in addition to those with Atg7. It will be interesting in the future to learn whether other E2-mediated steps in autophagy, such as during Atg12 Atg5 enhancement of Atg8 transfer from Atg3 to PE, also involve interactions near or at autophagy E2 backsides.

\section{Online methods}

\section{Constructs, peptides, and proteins for in vitro studies}

All sequences in this study are from the budding yeast, S. cerevisiae. Constructs were generated by standard methods and coding regions verified by automated sequencing. Atg7, Atg3, Atg3FR, Atg8 and variants were prepared as described ${ }^{21}$. Atg10 was expressed as a GST-fusion in RIL codon-enhanced BL21(DE3) (Agilent), purified by glutathione affinity, and following treatment with thrombin by anion exchange and gel filtration chromatography. For Atg7cis, His 6 -TEV-Atg7(R511E) and GST-thrombin- Atg7(289-630, 
C507A E524R), and for Atg7trans, His 6 -TEV-Atg7(C507A R511E) and GST-thrombinAtg7(residues 289-630, E524R) were coexpressed with the His 6 -fusion from pRSF-Duet the GST-fusion from pGEX-4T1.

For the Atg7-Atg3 crystals, Atg3(C41A C76A C83A) (Atg3Cys234only) and Atg7 (residues 1-613) were reduced with $10 \mathrm{mM}$ DTT and purified using an SD200 column in 20 mM HEPES (pH 7.0), $150 \mathrm{mM} \mathrm{NaCl}$. 0.5 mM Atg3Cys234only was mixed with 5x BisMaleimidoethane (BMOE, Pierce) on ice for $>1$ hour, followed by desalting (PD-10, GE) to remove unreacted crosslinker. $3 \mu \mathrm{M}$ Atg3Cys234only BMOE and $1.5 \mu \mathrm{M}$ Atg7(1-613) were mixed for 20 seconds, and the crosslinking was quenched with 50 mM DTT. Atg7(1613) BMOE Atg3Cys234only (referred to as Atg7-Atg3 in the main text) was purified by anion exchange and gel filtration into a final buffer of $50 \mathrm{mM}$ HEPES (pH 7.0), $150 \mathrm{mM}$ $\mathrm{NaCl}, 10 \mathrm{mM}$ DTT, concentrated to $12.5 \mathrm{mg} \mathrm{ml}^{-1}$, aliquoted, flash-frozen in liquid nitrogen and stored at $-80^{\circ} \mathrm{C}$. Atg10(C26S C137S) (Atg10Cys133only) crosslinked to Atg7(1-613 C39S C195S C375A) (referred to as Atg7-Atg10 in the main text) was prepared similarly, except BMOE was added to $\sim 0.2 \mathrm{mM}$ Atg10(C26S C137S); crosslinking was carried out with 1.2 $\mu \mathrm{M}$ Atg7(1-613 C39S C195S C375A) and $2.4 \mu \mathrm{M}$ Atg10Cys133only; the final buffer was $25 \mathrm{mM}$ HEPES (pH 7.0), $100 \mathrm{mM} \mathrm{NaCl}, 5 \mathrm{mM}$ DTT; and Atg7-Atg10 was concentrated to $7 \mathrm{mg} \mathrm{ml}^{-1}$.

Atg7NTD (K14A F16A D18A) was prepared as described ${ }^{21}$, except concentrated to $\sim 90 \mathrm{mg}$ $\mathrm{ml}^{-1}$ prior to crystallization.

\section{Crystallization, data collection, and structure determination}

Crystals of Atg7-Atg3 were grown by microseeding a room temperature sitting drop of $2 \mu 1$ Atg7(1-613) BMOE Atg3Cys234only with $1.4 \mu \mathrm{l}$ well solution of $100 \mathrm{mM}$ sodium citrate (pH 5.8), 13.5\% PEG4000, 10\% isopropanol, $5 \%$ dioxane, $88 \mathrm{mM}$ ammonium sulfate, and cryoprotected in well solution supplemented with $15 \%$ glycerol and $15 \%$ ethylene glycol prior to data collection at APS 24-ID-E $(\lambda=0.9792 \AA)$ at $100 \mathrm{~K}$. Crystals of Atg7-Atg10 were grown by microseeding a room temperature sitting drop of $400 \mathrm{nl}$ Atg7 (1-613, C39S C195S C375A) BMOE Atg10Cys133only in 25 mM HEPES (pH 7.0), $100 \mathrm{mM} \mathrm{NaCl}, 5$ $\mathrm{mM}$ DTT with $400 \mathrm{nl}$ well solution containing $76.5 \mathrm{mM} \mathrm{Na} / \mathrm{K}$ phosphate $(\mathrm{pH} 6.5), 9 \mathrm{mM}$ Tris (pH 8.5), $153 \mathrm{mM} \mathrm{NaCl}, 100 \mathrm{mM}$ glycine, $72 \mathrm{mM} \mathrm{Na} / \mathrm{K}$ tartrate, 19.125\% PEG1000, 0.045\% PEG5000MME, 4.5\% dioxane, and cryoprotected in well solution supplemented with $30 \%$ glycerol prior to data collection at APS 24-ID-C $(\lambda=0.9795 \AA)$ at $100 \mathrm{~K}$. Crystals of Atg7NTD (K14A F16A D18A) were grown at $4^{\circ} \mathrm{C}$ in $7.2 \%$ isopropanol, $50 \mathrm{mM}$ citrate buffer ( $\mathrm{pH} 4.1), 0.1 \mathrm{M}$ potassium thiocyanate, and cryoprotected in solution consisting of 75 $\mu \mathrm{l}$ well buffer mixed with $25 \mu \mathrm{l}$ isopropanol, prior to data collection at ALS 8.2.2 $(\lambda=0.979$ $\AA$ ) at $100 \mathrm{~K}$.

Data were processed with HKL2000 42 . Phases were determined by molecular replacement using Phaser ${ }^{43}$ with the following search models: Atg7-Atg3: Atg7NTD ${ }^{21}$, Atg7CTD ${ }^{21}$, Atg3 (lacking the FR) ${ }^{27}$; Atg7-Atg10: Atg7NTD ${ }^{21}$, Atg7CTD ${ }^{21}$, with Atg10 built manually into clear density through bootstrapping approaches; Atg7NTD (K14A F16A D18A): Atg7NTD ${ }^{21}$. The structures were completed by cycles of manual rebuilding using $\mathrm{Coot}^{44}$ and refinement using $\mathrm{FFX}^{45}$ and Phenix ${ }^{46}$. For both copies of Atg3 in the asymmetric unit, 
the first three turns of the HR helix were modeled into weak, but obviously helical, density for the backbone based on the prior structure of uncomplexed Atg $3^{27}$. Otherwise, several loops in Atg7, Atg3, and Atg10, and the BMOE crosslink not visible in the electron density were not modeled in the structures.

\section{In vitro biochemical and biophysical assays}

Assays for pulse-chase transfer of $\left[{ }^{32} \mathrm{P}\right] \mathrm{Atg} 8$ from Atg7 to Atg3, BMOE crosslinking between the single-cysteine version of Atg3 (Cys234only) and Atg7, and in vitro ligation of Atg8 to PE were performed as described previously ${ }^{21}$. Crosslinking assays were performed at a range of Atg7 and Atg3 (Cys234only) or Atg10 (a single cysteine version with Cys133only, with C26A and C137A) concentrations, for between 30-120 seconds, and results shown are similar to those obtained over a range of concentrations and times, on different days. Crosslinking experiments using the cis and trans versions of Atg7 in Fig. 2c contained $1 \mu \mathrm{M}$ Atg7 (cis or trans) and $2 \mu \mathrm{M}$ Atg3 or Atg10. Atg7xAtg3 crosslinking experiments detected by western blotting contained $60 \mathrm{nM}$ wild-type Atg7 and $120 \mathrm{nM}$ Atg3 (Cys234only). Reactions in Fig. 3f contained $60 \mathrm{nM}$ wild-type Atg7 and $120 \mathrm{nM}$ Atg10 (Cys133only); in Fig. 3g and 5b contained $150 \mathrm{nM}$ Atg7 and $300 \mathrm{nM}$ Atg10. Anti-Atg7 western blots of the crosslinking assays used \#SC8965 (Santa Cruz) at 1:250. The reaction forcing Atg8 onto Atg10 in Fig. 3d contained $1 \mu \mathrm{M}$ Atg7, 60 $\mu \mathrm{M}$ Atg8, $40 \mu \mathrm{M}$ Atg10, and $500 \mu \mathrm{M} \operatorname{Atg} 3^{\text {FRpep. }}$.

\section{Yeast media and growth conditions}

Yeast cells were grown in rich (YPD; $1 \%$ yeast extract, $2 \%$ peptone, and 2\% glucose) or synthetic minimal (SMD; $0.67 \%$ yeast nitrogen base, $2 \%$ glucose, and auxotrophic amino acids and vitamins as needed) media. For nitrogen starvation conditions, synthetic medium lacking nitrogen (SD-N; 0.17\% yeast nitrogen base without ammonium sulfate or amino acids, and $2 \%$ glucose) was used.

\section{Yeast plasmids and strains}

pCu414, pCuPA416 and pGFPATG8(405) have been reported previously ${ }^{4-49}$. To generate pCuATG3(414), the open reading frame (ORF) of wild-type or mutant ATG3 was amplified by PCR from the corresponding pGEX bacterial expression vector and cloned into the BamHI and SalI sites of pCu414. To generate pCuPAATG7(416), the open reading frame (ORF) of wild-type or mutant ATG7 was amplified by PCR from the corresponding pGEX bacterial expression vector and cloned into the ClaI and SalI sites of pCuPA416.

The yeast strains used in this study are KDM1222 [SEY6210 GFP-ATG8(405) atg3 $\Delta:: \mathrm{HIS} 3$, this study], KDM1223 [SEY6210 GFP-ATG8(405) atg7 $::$ HIS3, this study], KDM1225 [SEY6210 GFP-ATG8(405), this study], KDM1402 [SEY6210 pho13 $\Delta$ pho8 60 atg3 $\Delta::$ LEU2, this study], KDM1404 [SEY6210 pho13 $\Delta$ pho8 $\Delta 60$ atg7 $\Delta:: L E U 2$, this study], SEY6210 [MATa ura3-52 leu2-3,112 his3- $\Delta 200$ trp1- $\Delta 901$ lys2-801 suc2- $\Delta 9$ mel $\left.\mathrm{GAL}^{50}\right]$, and WLY176 [SEY6210 pho13 $\Delta$ pho8 $\left.\Delta 60^{51}\right]$.

For gene disruption, the entire coding regions were replaced with Kluyvermomyces lactis LEU2, and Saccharomyces kluyveri HIS3 genes using PCR primers containing 40 bases 
identical to the flanking regions of the open reading frames. To construct GFP-Atg8 strains, the integrative plasmid pGFPATG8(405) was linearized with AfIII and integrated into the LEU2 locus.

\section{Yeast assays}

The GFP-Atg8 processing and Pho8 860 assays to monitor autophagy progression were performed as previously described ${ }^{52}$. For the alkaline phosphatase assay, yeast were grown in either synthetic minimal SMD (+) or nitrogen starvation SD-N (-) media, as indicated. Two $\mathrm{A}_{600}$ equivalents of yeast cells were harvested and resuspended in $150 \mu \mathrm{l}$ lysis buffer (20 mM PIPES, $\mathrm{pH} 7.0,0.5 \%$ Triton X-100, $50 \mathrm{mM} \mathrm{KCl}, 100 \mathrm{mM}$ potassium acetate, 10 $\mathrm{mM} \mathrm{MgSO}_{4}, 10 \mu \mathrm{M} \mathrm{ZnSO}_{4}$, and $1 \mathrm{mM}$ PMSF). The cells were lysed by vortexing with glass beads for $5 \mathrm{~min}, 50 \mu \mathrm{l}$ of extract was added to $450 \mu \mathrm{l}$ reaction buffer $(250 \mathrm{mM}$ Tris$\mathrm{HCl}, \mathrm{pH} 8.5,0.4 \%$ Triton $\mathrm{X}-100,10 \mathrm{mM} \mathrm{MgSO}_{4}$, and $1.25 \mathrm{mM}$ nitrophenylphosphate), and samples were incubated for $\sim 15 \mathrm{~min}$ at $30^{\circ} \mathrm{C}$ before terminating the reaction by adding 500 $\mu \mathrm{l}$ of stop buffer ( $2 \mathrm{M}$ glycine, $\mathrm{pH} 11$ ). Production of nitrophenol was monitored by measuring the absorbance at $400 \mathrm{~nm}$ using a spectrophotometer (DU-640B; Beckman Coulter), and the nitrophenol concentration was calculated using Beer's law with $\varepsilon 400=$ $18,000 \mathrm{M}^{-1} \mathrm{~cm}^{-1}$. Protein concentration in the extracts was measured with the Pierce BCA assay (Thermo Fisher Scientific), and one activity unit was defined as nmol nitrophenol $/ \mathrm{min} / \mathrm{mg}$ protein. The activity for WT with empty vector was set to $100 \%$ and the other activities were normalized. Error bars (standard deviation) were obtained from three independent repeats. Pgk1 immunoblot loading controls are shown. Assays monitoring Atg8 PE formation and GFP-Atg8 localization in yeast were performed as previously described ${ }^{53,54}$. Antibodies for $\operatorname{Atg} 8^{55}$ and $\operatorname{Atg} 3^{56}$ were used at 1:3,000 and 1:2,000, respectively. GFP was detected with anti-YFP antibody JL-8 (Clontech \#632381) at 1:5000. Anti-Pgk1 was provided by J. Thorner (University of California, Berkeley, CA) and used at 1:500,000. Anti-PA tag western blots used \#Z0113 (Dako, Denmark; no longer commercially available) at 1:10,000.

\section{Supplementary Material}

Refer to Web version on PubMed Central for supplementary material.

\section{Acknowledgments}

This work was supported by ALSAC, the Howard Hughes Medical Institute, the St. Jude Cancer Center grant (5P30CA021765), NIH R01GM077053 to BAS and R01GM053396 to DJK. BAS is an Investigator of the Howard Hughes Medical Institute. We are grateful to C. Regni and A. Williams for help in early stages of this project, M. Frank and C. Rock (St. Jude Children's Research Hospital, Memphis, TN) for providing liposomes, and D.W. Miller, S. Bozeman, D.J. Miller, Y. Qiu, and J. Bollinger for administrative, computational or technical support. NECAT is supported by NIH NCRR RR-15301, APS by US DOE W-31-109-ENG-38, ALS by US DOE DEAC02-05CH11231.

\section{References}

1. Ohsumi Y, Mizushima N. Two ubiquitin-like conjugation systems essential for autophagy. Semin Cell Dev Biol. 2004; 15:231-6. [PubMed: 15209383]

2. Nakatogawa H, Suzuki K, Kamada Y, Ohsumi Y. Dynamics and diversity in autophagy mechanisms: lessons from yeast. Nat Rev Mol Cell Biol. 2009; 10:458-67. [PubMed: 19491929] 
3. Geng J, Klionsky DJ. The Atg8 and Atg12 ubiquitin-like conjugation systems in macroautophagy. EMBO Rep. 2008; 9:859-64. [PubMed: 18704115]

4. Kirkin V, et al. A role for NBR1 in autophagosomal degradation of ubiquitinated substrates. Mol Cell. 2009; 33:505-16. [PubMed: 19250911]

5. Lamark T, Kirkin V, Dikic I, Johansen T. NBR1 and p62 as cargo receptors for selective autophagy of ubiquitinated targets. Cell Cycle. 2009; 8:1986-90. [PubMed: 19502794]

6. Hanada T, et al. The Atg12-Atg5 conjugate has a novel E3-like activity for protein lipidation in autophagy. J Biol Chem. 2007; 282:37298-302. [PubMed: 17986448]

7. Suzuki K, et al. The pre-autophagosomal structure organized by concerted functions of $A P G$ genes is essential for autophagosome formation. EMBO J. 2001; 20:5971-81. [PubMed: 11689437]

8. Mizushima N, et al. Dissection of autophagosome formation using Apg5-deficient mouse embryonic stem cells. J Cell Biol. 2001; 152:657-68. [PubMed: 11266458]

9. Kuma A, Mizushima N, Ishihara N, Ohsumi Y. Formation of the approximately 350-kDa Apg12Apg5.Apg16 multimeric complex, mediated by Apg16 oligomerization, is essential for autophagy in yeast. J Biol Chem. 2002; 277:18619-25. [PubMed: 11897782]

10. Suzuki K, Ohsumi Y. Molecular machinery of autophagosome formation in yeast, Saccharomyces cerevisiae. FEBS Lett. 2007; 581:2156-61. [PubMed: 17382324]

11. Fujita N, et al. The Atg16L complex specifies the site of LC3 lipidation for membrane biogenesis in autophagy. Mol Biol Cell. 2008; 19:2092-100. [PubMed: 18321988]

12. Shintani T, Klionsky DJ. Autophagy in health and disease: a double-edged sword. Science. 2004; 306:990-5. [PubMed: 15528435]

13. Kundu M, Thompson CB. Autophagy: Basic principles and relevance to disease. Annual Review of Pathology-Mechanisms of Disease. 2008; 3:427-455.

14. Levine B, Kroemer G. Autophagy in the pathogenesis of disease. Cell. 2008; 132:27-42. [PubMed: 18191218]

15. Mizushima N, Levine B, Cuervo AM, Klionsky DJ. Autophagy fights disease through cellular selfdigestion. Nature. 2008; 451:1069-75. [PubMed: 18305538]

16. Schulman BA, Harper JW. Ubiquitin-like protein activation by E1 enzymes: the apex for downstream signalling pathways. Nat Rev Mol Cell Biol. 2009; 10:319-31. [PubMed: 19352404]

17. Walden H, et al. The structure of the APPBP1-UBA3-NEDD8-ATP complex reveals the basis for selective ubiquitin-like protein activation by an E1. Mol Cell. 2003; 12:1427-37. [PubMed: 14690597]

18. Walden H, Podgorski MS, Schulman BA. Insights into the ubiquitin transfer cascade from the structure of the activating enzyme for NEDD8. Nature. 2003; 422:330-4. [PubMed: 12646924]

19. Lois LM, Lima CD. Structures of the SUMO E1 provide mechanistic insights into SUMO activation and E2 recruitment to E1. EMBO J. 2005; 24:439-51. [PubMed: 15660128]

20. Lee I, Schindelin H. Structural insights into E1-catalyzed ubiquitin activation and transfer to conjugating enzymes. Cell. 2008; 134:268-78. [PubMed: 18662542]

21. Taherbhoy AM, et al. Atg8 transfer from Atg7 to Atg3: a distinctive E1-E2 architecture and mechanism in the autophagy pathway. Mol Cell. 2011; 44:451-61. [PubMed: 22055190]

22. Noda NN, et al. Structural basis of Atg8 activation by a homodimeric E1, Atg7. Mol Cell. 2011; 44:462-75. [PubMed: 22055191]

23. Hong SB, et al. Insights into noncanonical E1 enzyme activation from the structure of autophagic E1 Atg7 with Atg8. Nature structural \& molecular biology. 2011; 18:1323-30.

24. Olsen SK, Capili AD, Lu X, Tan DS, Lima CD. Active site remodelling accompanies thioester bond formation in the SUMO E1. Nature. 2010; 463:906-12. [PubMed: 20164921]

25. Huang DT, et al. Structural basis for recruitment of Ubc12 by an E2 binding domain in NEDD8's E1. Mol Cell. 2005; 17:341-50. [PubMed: 15694336]

26. Tokgoz Z, et al. E1-E2 interactions in ubiquitin and Nedd8 ligation pathways. J Biol Chem. 2012; 287:311-21. [PubMed: 22069333]

27. Yamada Y, et al. The crystal structure of Atg3, an autophagy-related ubiquitin carrier protein (E2) enzyme that mediates Atg8 lipidation. J Biol Chem. 2007; 282:8036-43. [PubMed: 17227760] 
28. Yamaguchi M, et al. Structural Insights into Atg10-Mediated Formation of the AutophagyEssential Atg12-Atg5 Conjugate. Structure. 2012; 20:1244-54. [PubMed: 22682742]

29. Noda T, Matsuura A, Wada Y, Ohsumi Y. Novel system for monitoring autophagy in the yeast Saccharomyces cerevisiae. Biochem Biophys Res Commun. 1995; 210:126-32. [PubMed: 7741731]

30. Shintani T, Klionsky DJ. Cargo proteins facilitate the formation of transport vesicles in the cytoplasm to vacuole targeting pathway. J Biol Chem. 2004; 279:29889-94. [PubMed: 15138258]

31. Ichimura Y, et al. A ubiquitin-like system mediates protein lipidation. Nature. 2000; 408:488-92. [PubMed: 11100732]

32. Mizushima N, et al. A protein conjugation system essential for autophagy. Nature. 1998; 395:3958. [PubMed: 9759731]

33. Tanida I, Tanida-Miyake E, Komatsu M, Ueno T, Kominami E. Human Apg3p/Aut1p homologue is an authentic E2 enzyme for multiple substrates, GATE-16, GABARAP, and MAP-LC3, and facilitates the conjugation of hApg12p to hApg5p. J Biol Chem. 2002; 277:13739-13744. [PubMed: 11825910]

34. Radoshevich L, et al. ATG12 Conjugation to ATG3 Regulates Mitochondrial Homeostasis and Cell Death. Cell. 2010; 142:590-600. [PubMed: 20723759]

35. Brzovic PS, Lissounov A, Christensen DE, Hoyt DW, Klevit RE. A UbcH5/ubiquitin noncovalent complex is required for processive BRCA1-directed ubiquitination. Mol Cell. 2006; 21:873-80. [PubMed: 16543155]

36. Capili AD, Lima CD. Structure and analysis of a complex between SUMO and Ubc9 illustrates features of a conserved E2-Ubl interaction. J Mol Biol. 2007; 369:608-18. [PubMed: 17466333]

37. Duda DM, et al. Structure of a SUMO-binding-motif mimic bound to Smt3p-Ubc9p: conservation of a non-covalent ubiquitin-like protein-E2 complex as a platform for selective interactions within a SUMO pathway. J Mol Biol. 2007; 369:619-30. [PubMed: 17475278]

38. Knipscheer P, van Dijk WJ, Olsen JV, Mann M, Sixma TK. Noncovalent interaction between Ubc9 and SUMO promotes SUMO chain formation. The EMBO J. 2007; 26:2797-807. [PubMed: 17491593]

39. Das R, et al. Allosteric activation of E2-RING finger-mediated ubiquitylation by a structurally defined specific E2-binding region of gp78. Mol Cell. 2009; 34:674-85. [PubMed: 19560420]

40. Li W, et al. Mechanistic insights into active site-associated polyubiquitination by the ubiquitinconjugating enzyme Ube2g2. Proceedings of the National Academy of Sciences of the United States of America. 2009; 106:3722-7. [PubMed: 19223579]

41. Hibbert RG, Huang A, Boelens R, Sixma TK. E3 ligase Rad18 promotes monoubiquitination rather than ubiquitin chain formation by E2 enzyme Rad6. Proceedings of the National Academy of Sciences of the United States of America. 2011; 108:5590-5. [PubMed: 21422291]

42. Otwinowski, Z.; Minor, W. Processing of X-ray Diffraction Data Collected in Oscillation Mode. In: Carter, CW.; Sweet, RM., editors. Methods in Enzymology, Macromolecular Crystallography, part A. 1997. p. 307-326.

43. McCoy AJ, et al. Phaser crystallographic software. J Appl Crystallogr. 2007; 40:658-674. [PubMed: 19461840]

44. Emsley P, Lohkamp B, Scott WG, Cowtan K. Features and development of Coot. Acta Crystallogr D Biol Crystallogr. 2010; 66:486-501. [PubMed: 20383002]

45. Fenn TD, Schnieders MJ. Polarizable atomic multipole X-ray refinement: weighting schemes for macromolecular diffraction. Acta crystallographica Section D, Biological crystallography. 2011; 67:957-65.

46. Adams PD, et al. PHENIX: a comprehensive Python-based system for macromolecular structure solution. Acta Crystallogr D Biol Crystallogr. 2010; 66:213-21. [PubMed: 20124702]

47. Labbe S, Thiele DJ. Copper ion inducible and repressible promoter systems in yeast. Methods in enzymology. 1999; 306:145-53. [PubMed: 10432452]

48. Kim J, Huang WP, Stromhaug PE, Klionsky DJ. Convergence of multiple autophagy and cytoplasm to vacuole targeting components to a perivacuolar membrane compartment prior to de novo vesicle formation. J Biol Chem. 2002; 277:763-73. [PubMed: 11675395] 
49. Geng J, Nair U, Yasumura-Yorimitsu K, Klionsky DJ. Post-Golgi Sec proteins are required for autophagy in Saccharomyces cerevisiae. Mol Biol Cell. 2010; 21:2257-69. [PubMed: 20444978]

50. Robinson JS, Klionsky DJ, Banta LM, Emr SD. Protein sorting in Saccharomyces cerevisiae: isolation of mutants defective in the delivery and processing of multiple vacuolar hydrolases. Molecular and cellular biology. 1988; 8:4936-48. [PubMed: 3062374]

51. Kanki T, et al. A genomic screen for yeast mutants defective in selective mitochondria autophagy. Mol Biol Cell. 2009; 20:4730-8. [PubMed: 19793921]

52. Yen WL, et al. The conserved oligomeric Golgi complex is involved in double-membrane vesicle formation during autophagy. J Cell Biol. 2010; 188:101-14. [PubMed: 20065092]

53. Klionsky DJ, Cuervo AM, Seglen PO. Methods for monitoring autophagy from yeast to human. Autophagy. 2007; 3:181-206. [PubMed: 17224625]

54. Cheong H, Klionsky DJ. Biochemical methods to monitor autophagy-related processes in yeast. Methods Enzymol. 2008; 451:1-26. [PubMed: 19185709]

55. Huang WP, Scott SV, Kim J, Klionsky DJ. The itinerary of a vesicle component, Aut7p/Cvt5p, terminates in the yeast vacuole via the autophagy/Cvt pathways. J Biol Chem. 2000; 275:5845-51. [PubMed: 10681575]

56. Kim J, Huang WP, Klionsky DJ. Membrane recruitment of Aut7p in the autophagy and cytoplasm to vacuole targeting pathways requires Aut $1 \mathrm{p}$, Aut2p, and the autophagy conjugation complex. $\mathrm{J}$ Cell Biol. 2001; 152:51-64. [PubMed: 11149920] 

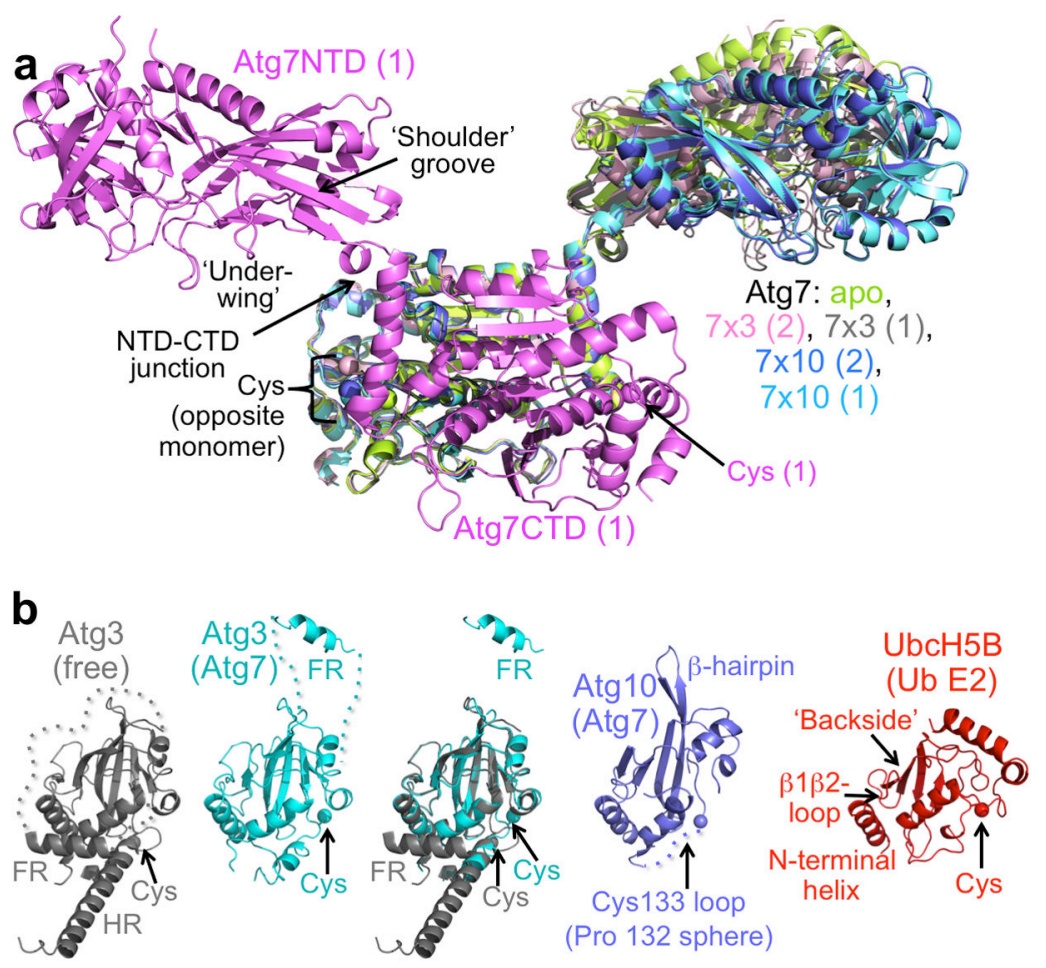

Figure 1. Overall structures of the autophagy E1 Atg7 and E2s Atg3 and Atg10

(a) Structure of Atg7 from the complex with Atg3 is shown in violet, indicating key structural features involved in E2 binding on one of the two protomers in the Atg7 homodimer. The adenylation active site and catalytic cysteine-containing CTD from the opposite protomer (pink) was superimposed with all other structures of $\operatorname{Atg} 7\left(\mathrm{apo}^{22}\right.$, 3VH2.pdb, green; protomer 1 from the crosslinked ("x") Atg7-Atg3 complex, grey; the two protomers from the crosslinked Atg7-Atg 10 complex, blue and light blue) showing different NTD-CTD orientations and catalytic cysteine locations among the structures. The two proteins from each dimer are indicated by (1) and (2).

(b) Crystal structures of unbound Atg $3^{27}$ (2DYT.pdb, grey), Atg7-bound Atg3 (cyan), Atg7bound Atg10 (slate), and the Ub E2 UbcH5B (2C4O.pdb, red) superimposed over their E2 core domains, indicating key structural features. Catalytic cysteine thiols are shown as spheres, except for Atg10, in which the cysteine loop (dashed lines) is not visible in the electron density, so a sphere marks the location of the alpha-carbon from the preceeding Pro132. 

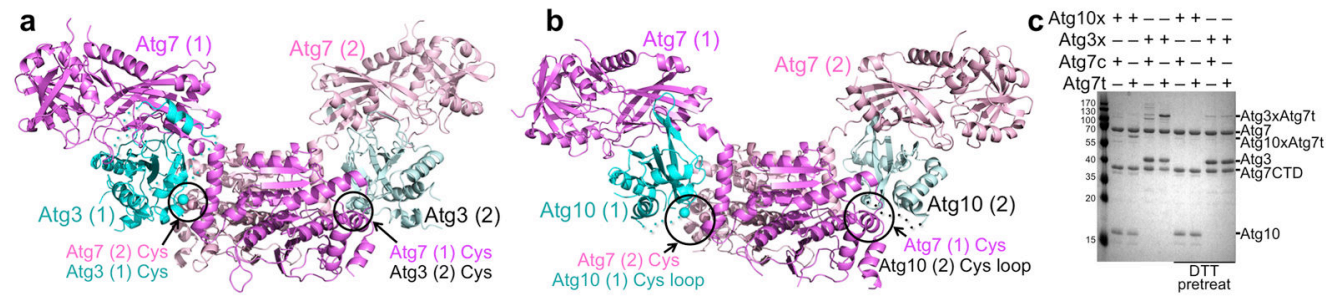

Figure 2. Overall trans architectures of autophagy E1-E2 complexes

(a) Structure of catalytic cysteine-to-cysteine crosslinked Atg7-Atg3 complex, with the two Atg7 protomers in the homodimer in violet and pink, the two Atg3 molecules in teal and cyan, and their catalytic cysteines shown as spheres. In the crystal, Atg3 binds

noncovalently to the NTD from one Atg7 protomer, and encircled is its catalytic cysteine approaching that from the opposite Atg7 in the homodimer. The encircled cysteines are crosslinked via BMOE. In (a) and (b), the two proteins from each dimer are indicated by (1) and (2).

(b) Structure of catalytic cysteine-to-cysteine crosslinked Atg7-Atg10 complex, with the two Atg7 protomers in the homodimer in violet and pink, the two Atg10 molecules in teal and cyan. Atg7 catalytic cysteines are shown as spheres, as is the alpha-carbon from Pro132 (molecule 1) and His131 (molecule 2) of Atg10. For the Atg10s, the catalytic Cys133 and loop are represented with dashed lines. In the crystal, Atg10 binds noncovalently to the NTD from one Atg7 protomer, and encircled is its catalytic cysteine loop approaching that from the opposite Atg7 in the homodimer. The encircled cysteines are crosslinked via BMOE. (c) Coomassie-stained SDS-PAGE showing bis-maleimidoethane (BMOE)-crosslinking between catalytic cysteine-only versions of Atg3 and Atg10 and cis (Atg7c) or trans (Atg7t) Atg7-Atg7CTD heterodimers. In Atg7c, only full-length Atg7 has an active site cysteine; in Atg7t, only the CTD has a catalytic cysteine. Control reactions were pre-treated with DTT to eliminate BMOE crosslinking. 


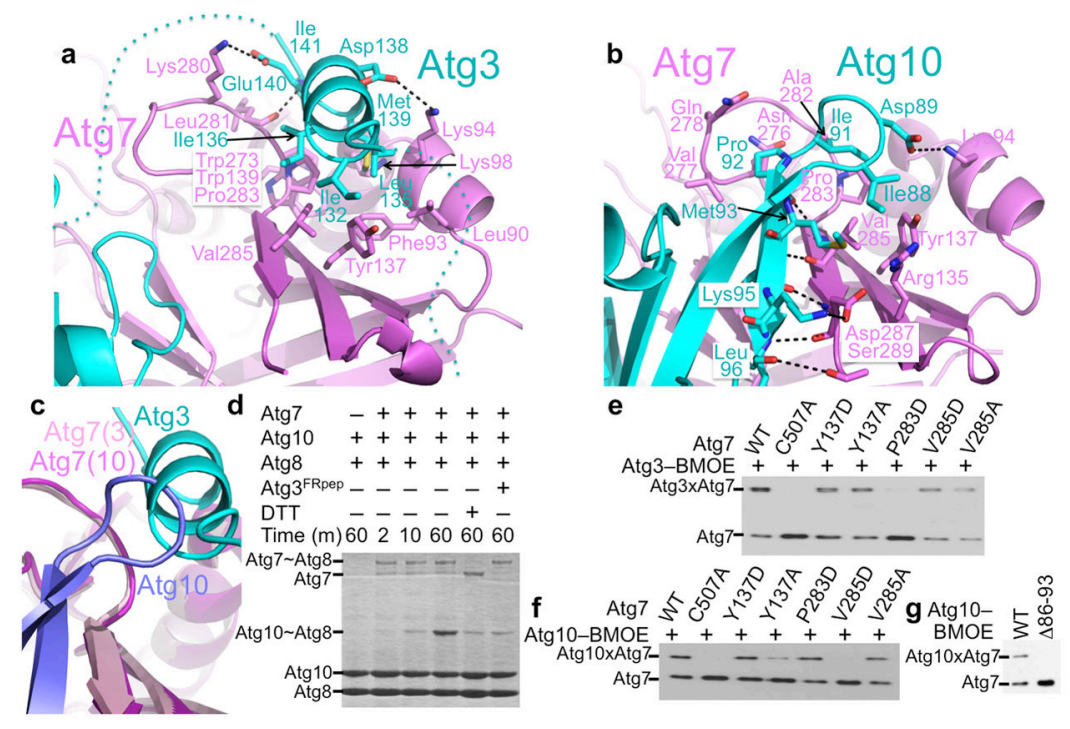

Figure 3. Atg7 NTD 'shoulder' groove interactions with autophagy E2s

(a) Close-up view of the Atg7 (violet) 'shoulder' goove interactions with helix from Atg3 FR (cyan). Loops not modeled in the Atg3 structure are shown as dotted lines.

(b) Close-up view of the Atg7 (violet) 'shoulder' goove interactions with the Atg10 $\beta$ hairpin (cyan).

(c) Close-up of shoulder grooves upon superimposing NTDs from Atg7 (pink and violet) in complexes with Atg3 and Atg10 (cyan and slate, respectively).

(d) Coomassie-stained SDS-PAGE showing artificial Atg8 conjugation to Atg10, in the absence or presence of a peptide corresponding to residues 130-142 from the Atg3 FR (Atg3 FRpep).

(e) Anti-Atg7 western blot showing BMOE crosslinking between a catalytic cysteine only version of Atg3 and the indicated 'shoulder' groove mutants of Atg7.

(f) Anti-Atg7 western blot showing BMOE crosslinking between a catalytic cysteine only version of Atg10 and the indicated 'shoulder' mutants of Atg7.

(g) Anti-Atg7 western blot showing BMOE crosslinking between a catalytic cysteine only version of Atg10 with otherwise wild-type sequence, or with residues 86-93 of the $\beta$-hairpin deleted, and Atg7. 

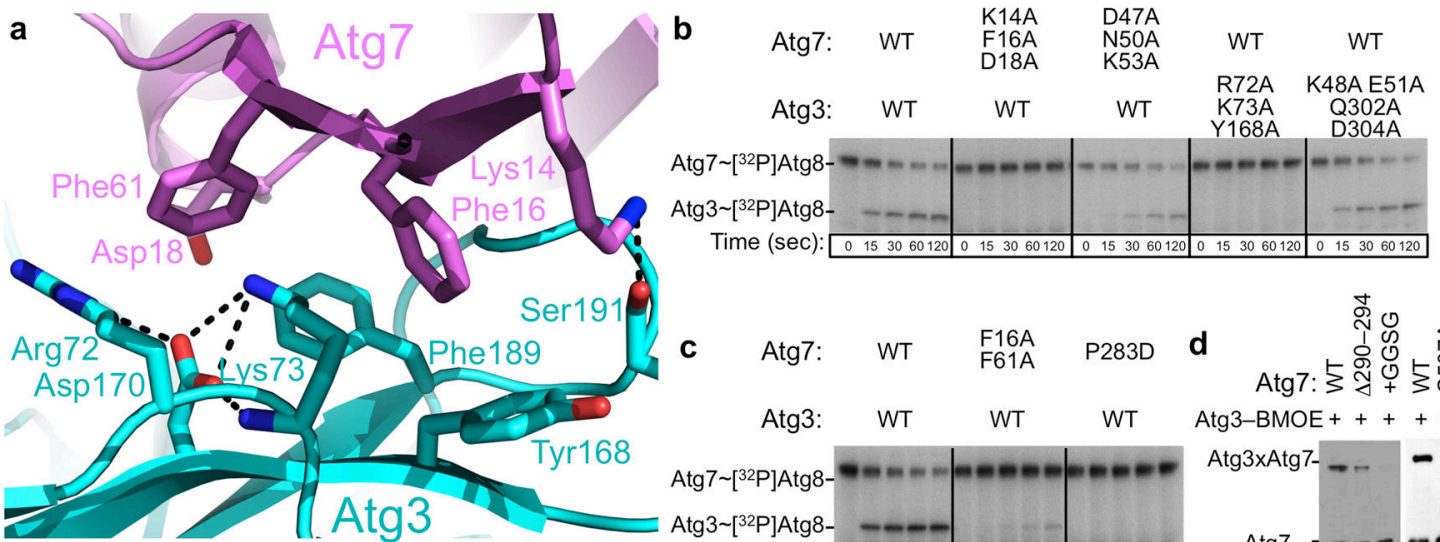

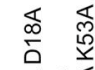
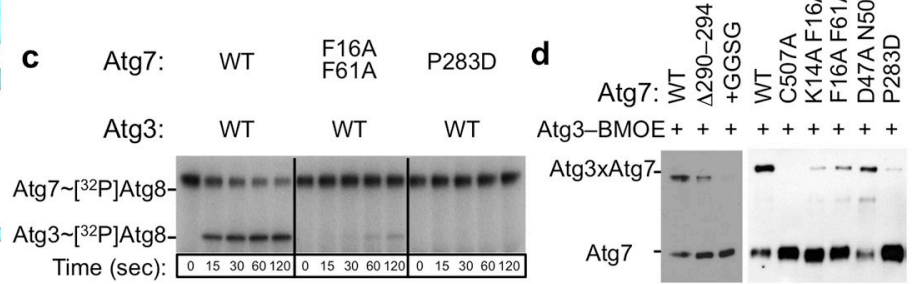

e



g

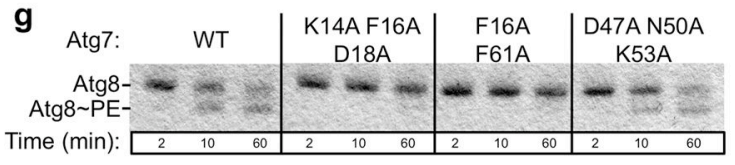

\section{i}

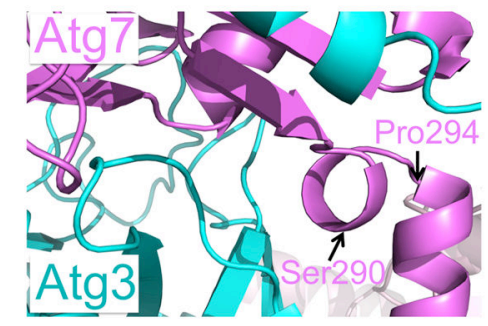

f

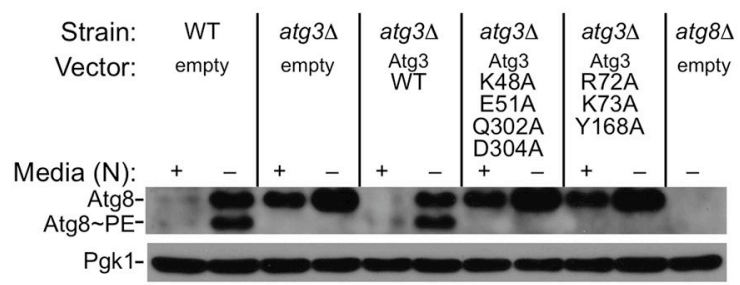

h

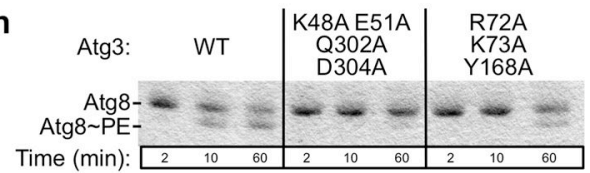

j

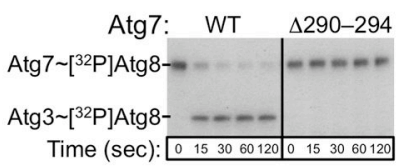

Figure 4. Atg7 'underwing' and NTD-CTD junction interactions with Atg3's backside and edge (a) Close-up of the Atg7 (violet) 'underwing' interactions with Atg3 (cyan) focused on the central hydrophobic cluster at the heart of the interaction. Interacting side chains are shown as sticks with oxygens in red and nitrogens in blue, with electrostatic interactions noted with dashed lines.

(b,c) Autoradiograms showing time course of pulse-chase $\left[{ }^{32} \mathrm{P}\right] \mathrm{Atg} 8$ transfer from wild-type or mutant versions of Atg7 to wild-type or backside mutant versions of Atg3.

(d) Immunoblot analysis of BMOE crosslinking between a catalytic cysteine only version of Atg3 and the indicated 'underwing' mutants of Atg7, or mutants with a Gly-Gly-Ser-Gly insertion after after Atg7's Leu291 or deletion of residues 290-294 at the NTD-CTD junction.

(e) Immunoblot analysis of Atg8 lipidation in wild-type or atg7 $\Delta$ yeast expressing PAtagged (P-) wild-type Atg7 or mutants. 
(f) Immunoblot analysis of Atg8 lipidation in wild-type or atg3 3 yeast strains harboring vectors expressing wild-type Atg3 or mutants.

(g) Coomassie-stained urea SDS-PAGE showing in vitro Atg8 lipidation in assays with wild-type or the indicated 'underwing' mutant versions of Atg7.

(h) Coomassie-stained urea SDS-PAGE showing in vitro Atg8 lipidation in assays with wild-type or the indicated 'backside' and 'distal edge' mutant versions of Atg3.

(i) Close-up of the Atg7 (violet) NTD-CTD junction interaction with Atg3 (cyan).

(j) Autoradiogram showing time course of pulse-chase [ $\left.{ }^{32} \mathrm{P}\right] \mathrm{Atg} 8$ transfer to Atg3 from wild-type Atg7 or a version with the NTD-CTD junction (residues 290-294) deleted. 

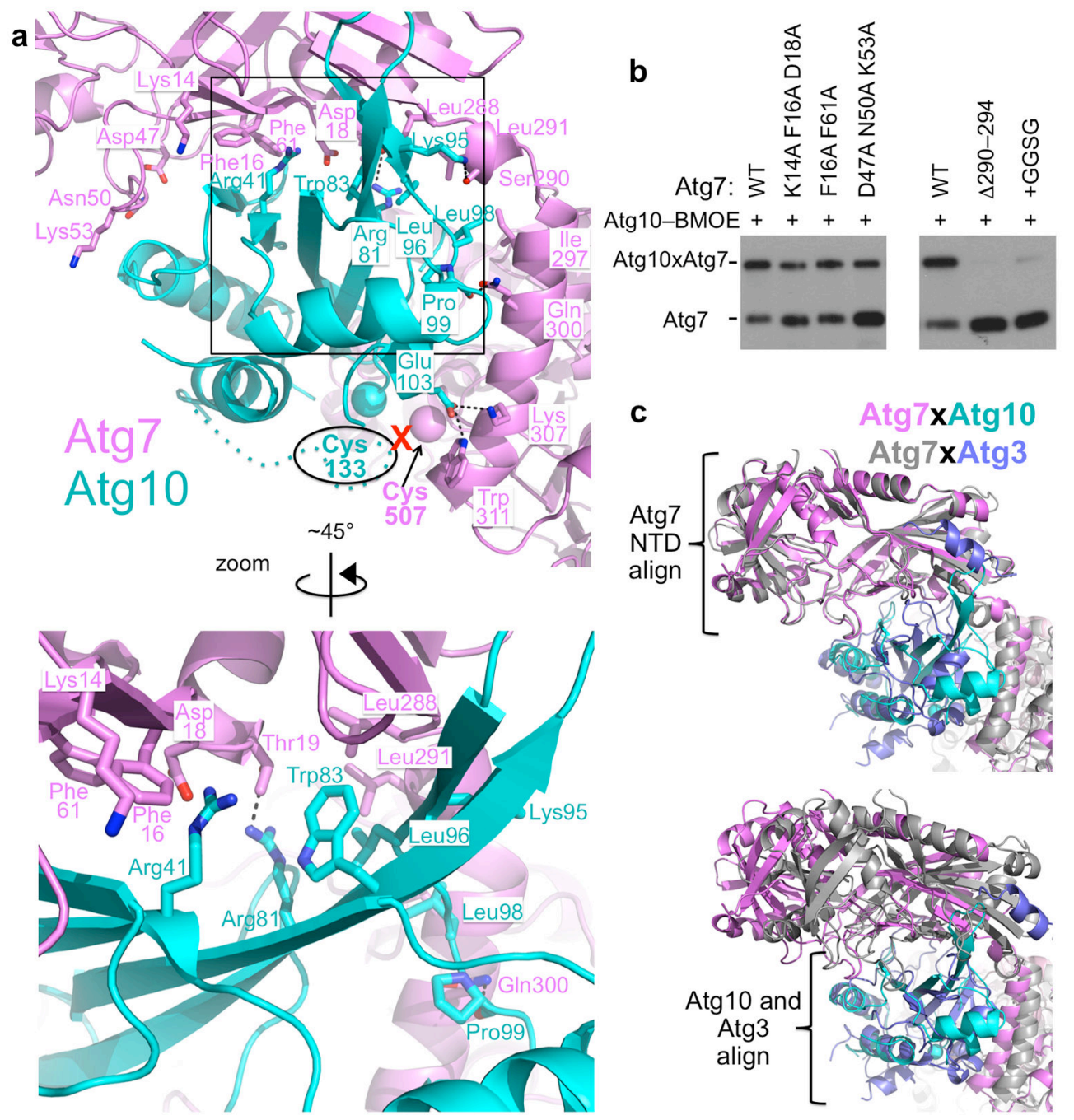

Figure 5. Atg7 'underwing' and NTD-CTD junction interactions with Atg10's backside and edge (a) Top, close-up of the Atg7 (violet) underwing and NTD-CTD junction interactions with $\operatorname{Atg} 10$ (cyan), with zoomed in view of the interactions emanating from the $\beta$-hairpin shown below rotated $\sim 45^{\circ}$ around the $y$-axis. Interacting side chains are shown as sticks with oxygens in red and nitrogens in blue, with electrostatic interactions noted with dashed lines. Red "x" denotes BMOE crosslink between Atg7 Cys507 and Atg10 Cys133.

(b) Anti-Atg7 western blot showing BMOE crosslinking (" $\mathrm{x}$ ”) between a catalytic cysteine only version of Atg10 and the indicated 'underwing' (left) mutants of Atg7, or versions with a Gly-Gly-Ser-Gly insertion after Atg7's Leu291 or deletion of residues 290-294 at the NTD-CTD junction (right).

(c) Structures of the Atg7 complexes crosslinked ("x") to Atg10 (violet and cyan) or to Atg3 (grey and slate) superimposed on either the Atg7 NTD (top) or over the Atg10 and Atg3 E2 core domains (bottom). 

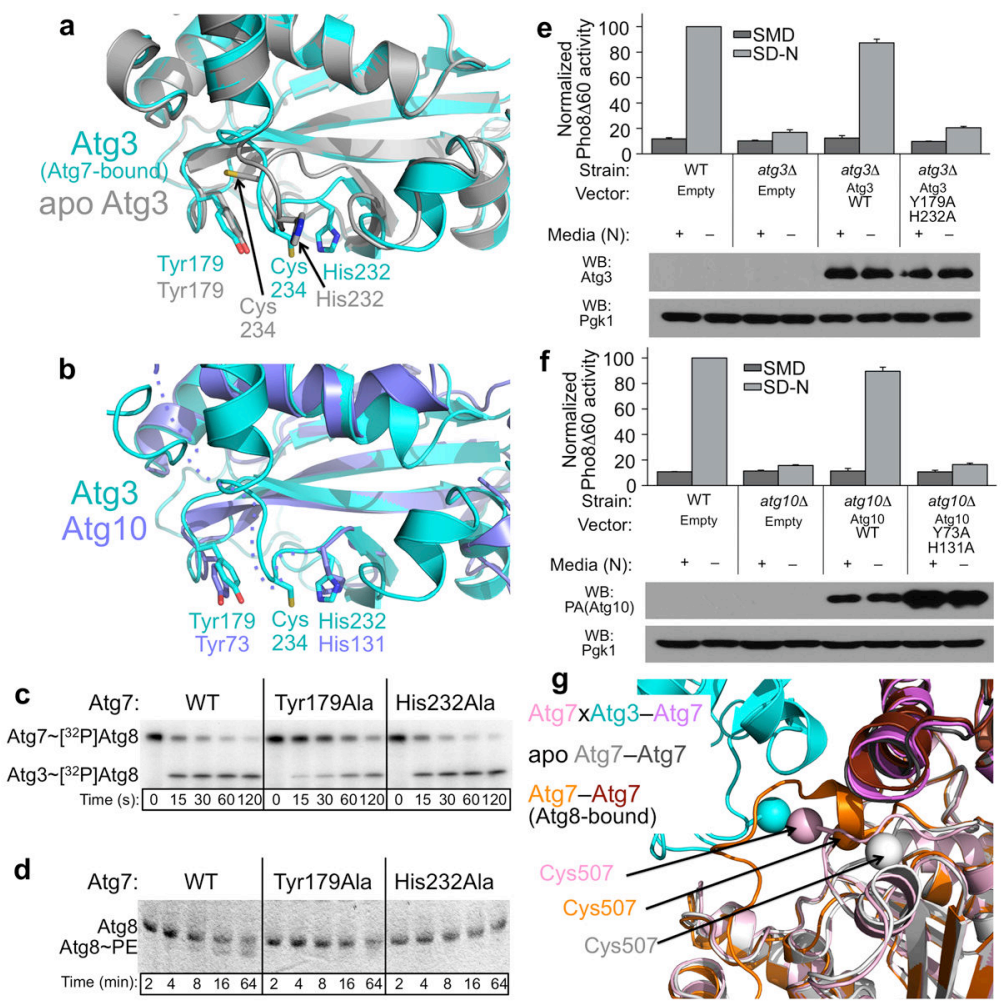

Figure 6. The Atg7, Atg3, and Atg10 catalytic cysteine-loops: autophagy E1 and E2 active site conformational variability

(a) Superposition of prior structure of Atg3 alone (2DYT.pdb, grey) ${ }^{27}$ and Atg7-bound Atg3 reveal reorientation of the catalytic cysteine-loop. Atg7 is not shown for simplification.

(b) Superposition of Atg7-bound Atg3 (cyan) and Atg10 (slate) reveals common positions for conserved tyrosine and histidine residues.

(c) Autoradiograms showing time course of $\left[{ }^{32} \mathrm{P}\right]$ Atg8 transfer from Atg7 to wild-type or Y179A or H232A mutant versions of Atg3.

(d) Coomassie-stained urea SDS-PAGE showing in vitro Atg8 lipidation in assays containing wild-type or Y179A or H232A mutant versions of Atg3.

(e) Pho8 $\Delta 60$ activity monitored in wild-type or atg $3 \Delta$ yeast harboring vectors expressing wild-type Atg3 or mutants. Error bars (standard deviation) were obtained from three independent repeats.

(f) Pho8 $\Delta 60$ activity (nonspecific autophagy) monitored in wild-type or atg $10 \Delta$ yeast strains harboring vectors expressing PA-tagged wild-type Atg10 or mutants. Error bars (standard deviation) were obtained from three independent repeats.

(g) Superposition of the adenylation domains from prior structures ${ }^{21-23}$ of unbound Atg7 (3VH2.pdb) or Atg8-bound Atg7CTD (3RUI.pdb), and the crosslinked ("x") Atg7-Atg3 complex described here, with the two Atg7 protomers colored in light and dark grey, orange and brown, and pink and violet, respectively. Atg3 is shown in cyan. Catalytic cysteine sulfur atoms (or an oxygen in 3RUI.pdb, which contains a C507S mutation) are shown as spheres. 


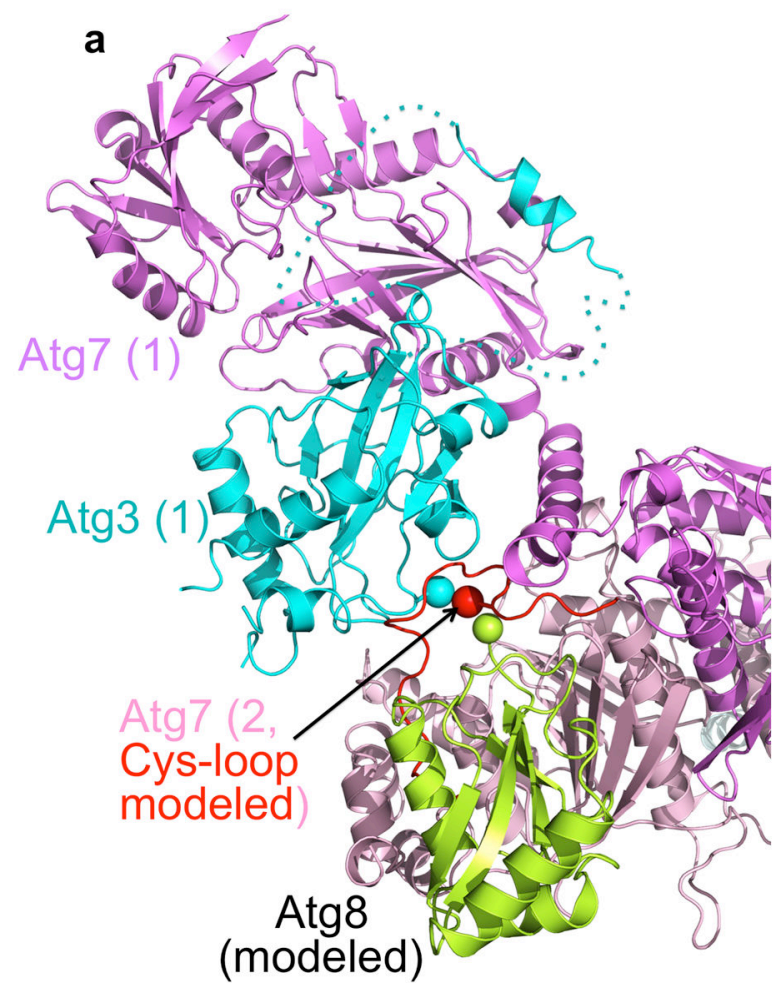

b

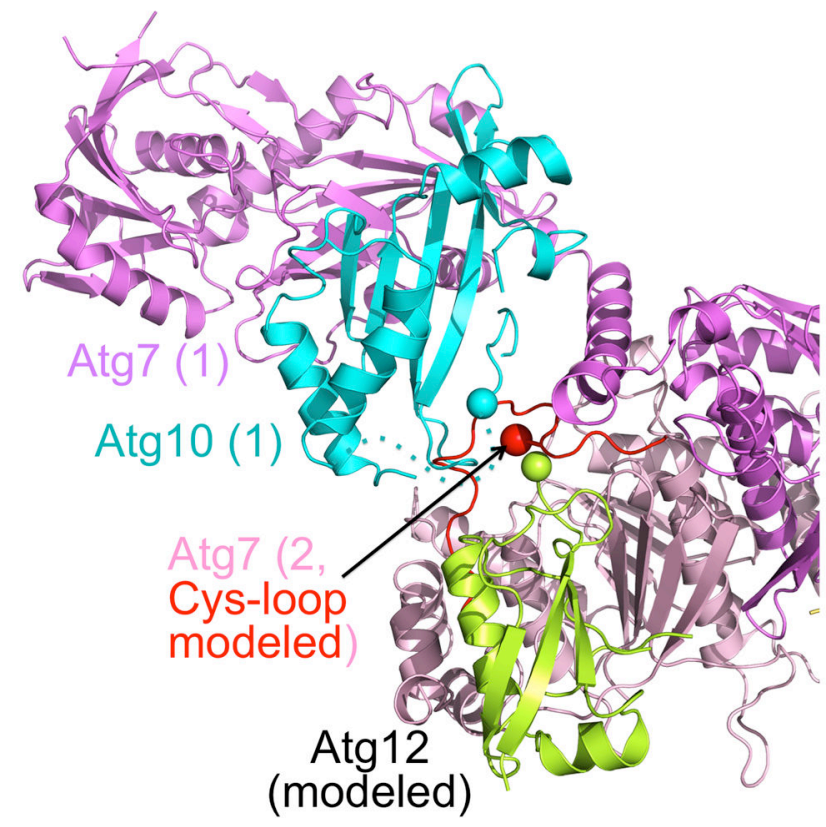

Figure 7. Models of autophagy Ubl-E1-E2 complexes

(a) Model of portion of homodimeric Atg7 (violet and pink, with cysteine-loop in red and catalytic cysteine sulfur a sphere) thioester-linked to Atg8 (lime, with C-terminus shown as sphere) bound to Atg3 (cyan, with catalytic cysteine sulfur a sphere). Loops in Atg3 not visible in the complex with Atg7 are shown as dashed lines.

(b) Model of portion of homodimeric Atg7 (violet and pink, with cysteine-loop in red and catalytic cysteine sulfur a sphere) thioester-linked to Atg12 (lime, with C-terminus shown as sphere) bound to Atg10 (cyan, with Ca from Pro132 preceding the catalytic Cys133 a sphere). The catalytic cysteine loop in Atg10 not visible in the complex with Atg7 is shown as a dashed line. 
Table 1

Data collection and refinement statistics (molecular replacement)

\begin{tabular}{|c|c|c|c|}
\hline & Atg7-BMOE-Atg3 & Atg7-BMOE-Atg10 & Atg7NTD (K14A F16A D18A) \\
\hline \multicolumn{4}{|l|}{ Data collection } \\
\hline Space group & $\mathrm{C} 2$ & $\mathrm{P} 2{ }_{1} 2_{1} 2$ & $\mathrm{P} 2{ }_{1} 2_{1} 2$ \\
\hline \multicolumn{4}{|l|}{ Cell dimensions } \\
\hline$a, b, c(\AA)$ & $281.6,125.1,71.1$ & $120.6,146.2,108.4$ & $56.8,74.6,76.5$ \\
\hline$\left.a, \beta, \chi^{\circ}\right)$ & $90,103.3,90$ & $90,90,90$ & $90,90,90$ \\
\hline Resolution $(\AA)$ & $40-2.7(2.8-2.7)$ & $50-2.9(3.0-2.9)$ & $50-1.7(1.76-1.70)$ \\
\hline$R_{\text {sym }}$ or $R_{\text {merge }}$ & $0.08(0.56)$ & $0.13(0.77)$ & $0.06(0.32)$ \\
\hline$I / \sigma I$ & $17.8(1.8)$ & $9.9(1.6)$ & $46.4(3.0)$ \\
\hline Completeness (\%) & $99.5(99.0)$ & $99.1(99.3)$ & $96.3(77.4)$ \\
\hline Redundancy & $4.1(4.0)$ & $3.2(3.3)$ & $6.5(4.0)$ \\
\hline \multicolumn{4}{|l|}{ Refinement } \\
\hline Resolution $(\AA)$ & $37-2.7$ & $50-2.9$ & $46-1.7$ \\
\hline No. reflections & 64763 & 42709 & 35168 \\
\hline$R_{\text {work }} / R_{\text {free }}$ & $0.193 / 0.246$ & $0.236 / 0.286$ & $0.176 / 0.207$ \\
\hline \multicolumn{4}{|l|}{ No. atoms } \\
\hline Protein & 12507 & 11423 & 2329 \\
\hline Ligand/ion & 2 & 2 & 0 \\
\hline Water & 43 & 0 & 257 \\
\hline \multicolumn{4}{|l|}{$B$-factors } \\
\hline Protein & 70.62 & 79.67 & 29.72 \\
\hline Ligand/ion & 80.23 & 90.41 & - \\
\hline Water & 45.88 & - & 38.55 \\
\hline \multicolumn{4}{|l|}{ R.m.s. deviations } \\
\hline Bond lengths $(\AA)$ & 0.009 & 0.004 & 0.006 \\
\hline Bond angles $\left({ }^{\circ}\right)$ & 1.214 & 0.842 & 1.005 \\
\hline
\end{tabular}

One crystal was used per data set. Values in parentheses are for highest-resolution shell. 